\title{
Dégradation De La Fertilité Des Sols Et De L'environnement Dans La Région Des Savanes Au Nord-Togo : Analyse Des Perceptions Et Stratégies D’adaptation Indigènes
}

\section{Magamana Abalo-Esso}

Doctorant en Géoressources; Université Joseph Ki-Zerbo, Ouaga I (UJKZOI), Burkina-Faso

\section{Gadedjisso-Tossou Agossou, PhD}

Chercheur au Centre d'Action pour la Sécurité Alimentaire et Développement Durable et la Valorisation des Ressources (CASADD-VR), Sokodé, Togo

\section{Blavet Didier}

Chercheur, agropédologue, Institut de Recherche pour le Développement (IRD), UMR Eco\&Sols Ecologie Fonctionnelle et biogéochimie des sols et des agrosystèmes, France

\section{Hien Edmond (Professeur titulaire)}

Université Joseph Ki-Zerbo, Ouaga I (UJKZ-OI) - UFR Sciences de la Vie et de la Terre, Président de l’Université de Fada N’Gourma, Burkina Faso.

\section{Chotte Jean Luc}

Directeur de Recherche au sein de l'UMR ECO\&SOLS, Président du huitième Comité Scientifique Francais de la Désertification (CSFD), Directeur de la Mission pour la Promotion de l'interdisciplinarité et de l'intersectorialité à , l'Institut de Recherche pour le Développement (IRD), France

\section{Doi:10.19044/esj.2021.v17n25p40}

Submitted: 29 March 2021

Accepted: 06 July 2021

Published: 31 July 2021
Copyright 2021 Author(s)

Under Creative Commons BY-NC-ND 4.0 OPEN ACCESS

Cite As:

Magamana A-E., Gadedjisso-Tossou A., Blavet D., Hien E. \& Chotte J.L. (2021). Dégradation De La Fertilité Des Sols Et De L'environnement Dans La Région Des Savanes Au Nord-Togo : Analyse Des Perceptions Et Stratégies D'adaptation Indigènes. European Scientific Journal, ESJ, 17(25), 40. https://doi.org/10.19044/esj.2021.v17n25p40 


\section{Résumé}

Le nord-ouest du Togo, est caractérisé par une forte pression sur les sols, entrainant une régression de parcs agroforestiers et une diminution rendements agricoles. Dans un tel milieu, l'appréciation des producteurs de ces changements ainsi que les méthodes qu'ils adoptent pour y faire face restent moins connus. C'est pour cet objectif qu'une enquête socioéconomique a été menée auprès des producteurs pour évaluer leurs perceptions sur la dégradation des sols et les changements climatiques afin d'identifier puis retenir les pratiques originales d'adaptation pouvant faire objet de diffusion. L’enquête menée sur un échantillon raisonné de 216 producteurs des deux sexes a montré que : 98,10\% des enquêtés sont conscients de la dégradation de leurs sols tandis que 99,50\% ressentent les effets des changements climatiques qui se manifestent en termes de longues sécheresses, de retards de pluies, de pluies violentes, abondantes et variable et d’augmentation de température. Les causes des changements évoquées par les producteurs sont : les pluies diluviennes (26,85\%), les mauvaises pratiques culturales (62,50\%), l'intensification agricole (46,76\%), le surpâturage (2,78\%), l'usage abusif de pesticides (20,37\%), l'augmentation de la population $(12,96 \%)$, la déforestation $(21,76 \%)$ et les feux de brousse $(6,48 \%)$. Globalement, les 98,6\% des enquêtés ont abandonné 63 ha de sol devenus irrécupérables. Les producteurs adoptent des mesures d'adaptation telles que l'apport de fumure organique, les rotations et associations culturales, la construction de bandes enherbées, de cordons pierreux et de diguettes, et le reboisement pour protéger et restaurer leurs champs. Plusieurs espèces de plantes désirées par les producteurs pour leur vertu sont en voie de disparition. La perception par les paysans des cas de longue sécheresse sont expliqués par les variables « âge du paysan », « la pratique de l'élevage », « l'accès aux services de vulgarisation » et « la pratique de l'agriculture ». Il est nécessaire de prendre ces variables en compte dans les projets et programmes de luttes contre la sécheresse dans le milieu. Les variables " âge du paysan », " mauvaises pratiques culturales », et « déforestation » influençant également la perception des paysans de dégradation des sols, doivent être également considérés dans les programmes et projets de restauration des sols dégradés dans le milieu. Il est important d'étudier les effets de ces pratiques sur la restauration des sols et le rendement des cultures.

Mots clés : Région Des Savanes, Dégradation De Sols, Pratiques Culturales 


\section{Degradation Of Soil Fertility And The Environment In The Savannah Region Of Northern Togo: Perception Analysis And Indigenous Adaptation Strategies}

\section{Magamana Abalo-Esso}

Doctorant en Géoressources; Université Joseph Ki-Zerbo, Ouaga I (UJKZOI), Burkina-Faso

\section{Gadedjisso-Tossou Agossou, PhD}

Chercheur au Centre d'Action pour la Sécurité Alimentaire et Développement Durable et la Valorisation des Ressources (CASADD-VR), Sokodé, Togo

\section{Blavet Didier}

Chercheur, agropédologue, Institut de Recherche pour le Développement (IRD), UMR Eco\&Sols Ecologie Fonctionnelle et biogéochimie des sols et des agrosystèmes, France

\section{Hien Edmond (Professeur titulaire)}

Université Joseph Ki-Zerbo, Ouaga I (UJKZ-OI) - UFR Sciences de la Vie et de la Terre, Président de l’Université de Fada N’Gourma, Burkina Faso.

\section{Chotte Jean Luc}

Directeur de Recherche au sein de l'UMR ECO\&SOLS, Président du huitième Comité Scientifique Francais de la Désertification (CSFD), Directeur de la Mission pour la Promotion de l'interdisciplinarité et de l’intersectorialité à ,l'Institut de Recherche pour le Développement (IRD), France

\section{Abstract}

The north-west of Togo is characterized by strong pressure on the soil, leading to a decline in agroforestry parks and a reduction in agricultural yields. In such an environment, producers' appreciation of these changes and the methods they adopt to deal with them remain less well known. It is for this purpose that a socioeconomic survey was conducted among producers to assess their perceptions of soil degradation and climate change to identify and then retain original adaptation practices that can be disseminated. The survey carried out on a purposely selected sample of 216 producers from both sexes. The results showed that: $98,10 \%$ of respondents are aware of the degradation of their soils while 99,50\% feel the effects of climate change which are manifested as long droughts, delayed rains, heavy rains and temperature rise. The causes of the changes mentioned by the producers are: torrential rains (26,85\%), bad cultivation practices (62,50\%), agricultural intensification (46,76\%), overgrazing (2,78\%) , the abusive use of pesticides (20,37\%), the increase in population (12,96\%), deforestation $(21,76 \%)$ and bush fires 
(6,48\%). Globally, 98,6\% of those surveyed abandoned 63 ha of land that had become irrecoverable. Producers adopt resilience measures such as providing organic manure, crop rotations and associations, the establishment of grass strips and stone bunds, bunds and reforestation to protect and restore their plots. Producers adopt adaptation measures such as adding organic manure, crop rotations and associations, construction of grass strips, stone bunds, bunds, and reforestation to protect and restore their fields. Several species of plants desired by producers for their virtue are endangered. The farmers' perception of cases of long drought are explained by the variables "age of the farmer", "the practice of animal husbandry", "access to extension services" and "the practice of agriculture". It is necessary to take these variables into account in projects and programs to fight against drought in the area. The variables "farmer's age «,» bad cultivation practices" and "deforestation" also influencing farmers' perception of soil degradation, must also be considered in programs and projects for the restoration of degraded soils in the area. It is important to study the effects of these practices on soil restoration and crop yields.

Keywords: Savannah Region, Soil Degradation, Cultivation Practices

\section{Introduction}

Le Togo est un petit pays de l'Afrique occidentale situé entre 6 et $11^{\circ}$ de latitude nord et 0 et $1^{\circ} 40$ de longitude. Il couvre une superficie de 56600 $\mathrm{km}^{2}$ et est limité au nord par la république du Burkina Faso, au sud par l'océan atlantique, à l'est par le République du Bénin et à l'ouest par la République du Ghana. Le pays est subdivisé en cinq régions administratives dont (du sud au nord) : la région Maritime, la région des Plateaux, la région Centrale, la région de la Kara et la région des Savanes.

Les terres cultivées sont estimées à près de 3,4 millions d'hectares (64\% du territoire) mais 55\% étaient cultivées en 2010. Avec une pluviométrie moyenne variant entre 800 et $1400 \mathrm{~mm}$ et une température moyenne de 27 à $28^{\circ} \mathrm{C}$, le Togo comptait 6191155 habitants en 2010 (4ème RGPH, 2010) et cette population devrait atteindre 7531928 habitants en 2017 avec un taux de croissance de $2,84 \%$ par an.

Les formations végétales sont fortement dégradées et le taux de déboisement est de l'ordre de $15000 \mathrm{ha} /$ an contre un rythme de reboisement qui ne dépasse guère 3000 ha annuellement (MERF, 2015). L'accroissement de la population exerce une forte pression sur les sols et l'environnement. Les principales causes de dégradation d'ordre environnemental et édaphique sont, entre autres, la déforestation ou le déboisement les incendies, le surpaturage, la dégradation des sols et le déclin de leur fertilité, la dégradation des aires protégées et de la faune, l'envasement des cours d'eau et de la lagune, la 
contamination et la dégradation de la qualité des eaux douces et la dégradation du cadre de vie. Un premier état de dégradation des sols et de l'environnement fut fait par Brabant en 1994; ainsi avait-il montré une relation entre la densité des cultures, la diminution de la jachère, la réduction du couvert végétal et le niveau de dégradation, dans un environnement bien déterminé. Par ailleurs, une étude menée en 1994 par l'Office de la Recherche Scientifique et Technique Outre-Mer (ORSTOM) et l'Institut National des Sols (INS) fait également ressortir en substance l'état de dégradation des terres au Togo. Cette étude avait mentionné trois secteurs de dégradation au Togo : les terres de barre dans Sud-est, le secteur du nord-est de Kandé et le secteur situé entre Dapaong et le Burkina-Faso (Brabant et al., 1994). Les simulations faites à cette époque, faisaient état d'une progression des terres dégradées qui passeront de $2 \%$ du territoire togolais à plus de $45 \%$ sur une période de 40 ans (entre 1995 et 2035). Le secteur de Dapaong était le plus dégradé avec divers types d'érosion : érosion en nappe et en rigole. Plus de 20 ans après cette étude, il est noté une évolution des superficies dégradées.

Malgré les programmes de reboisement et de lutte contre le déboisement force est de constater que le rythme de déboisement reste supérieur à celui du reboisement. Le taux de déforestation (89\% des superficie de la région des Savanes) est le plus élevé comparé aux autres régions du pays. A cela s'ajoute l'adoption timide de bonnes pratiques culturales vulgarisées par les services étatiques et les organisations non gouvernementales qui sensibilisent les producteurs depuis quelques années. Ces sensibilisations ont convaincu quelques producteurs qui reboisent et mettent en place des cordons pierreux et des bandes enherbées dans certains cantons afin de limiter les effets de la dégradation. Cependant, peu de plants survivent à la fin des projets dont la durée moyenne se situe entre 3 et 5 ans. Cette faible survie des plants serait imputable à la divagation incontrôlée des animaux en saison sèche, décimant les jeunes poussent d'arbres reboisés ; ce phénomène concerne $80 \%$ des villages dans la région des Savanes (RNA, 2013). Ainsi, les impacts de nombreux projets de développement ne sont pas durables, occasionnant le gaspillage de fonds, ce qui conduit à un éternel recommencement. La dégradation des sols et de l'environnement pourrait se poursuivre si les causes profondes de ce comportement ne sont pas bien cernées afin de mieux réorienter les actions et projets dans cette zone.

L'objectif global de cette étude est de contribuer à l'amélioration de la fertilité des sols et à la protection de l'environnement dans la région des Savanes au Togo. Il s'agit d'identifier les usages des terres ou les pratiques culturales effectuées dans la zone, ainsi que les contraintes rencontrées par les producteurs dans la mise en œuvre de ces pratiques à travers une enquête dans laquelle il sera notamment examiné "des pratiques originales ou 
traditionnelles mises en œuvre par les agriculteurs, et qui seraient susceptibles d'être améliorées afin de préserver ou augmenter les stocks de carbone"

Les objectifs spécifiques de l'étude sont: : (i) Evaluer la perception des producteurs sur dégradation des sols, les changements climatiques et leurs conséquences; (ii) Déterminer les motifs/causes d'adoption de pratiques de dégradation des sols et de l'environnement dans la zone; (iii) Déterminer les pratiques agro écologiques employées par les producteurs dans la zone; et (iv).

\section{Matériel et méthodes}

\section{Zone d'étude}

L’étude a été menée au nord-ouest de la région des Savanes couvrant une superficie de $4157 \mathrm{~km}^{2}$ sur $8470 \mathrm{~km}^{2}$ pour la région. C'est l'une des zones les plus dégradés du territoire togolais (Brabant et al., 1994). Les préfectures se situant dans la zone la plus dégradée et la plus peuplée de la région ont été choisis. Il s'agit des préfectures de Tone, de Cinkassé et de Tandjoare dont les densités de population sont respectivement de 234 habitants $/ \mathrm{km}^{2}, 268$ habitants $/ \mathrm{km}^{2}$ et de 138 habitants $/ \mathrm{km}^{2}$ contre 86 habitants $/ \mathrm{km}^{2}$ pour le Kpendjal, de 44 habitants $/ \mathrm{km}^{2}$ pour l'Oti et 98 habitants $/ \mathrm{km}^{2}$ pour la région des Savanes (RGPH, 2010). Le taux d'occupation des sols des trois préfectures pour les divers besoins (agriculture, habitations...) est de plus de 80\% (FAO, 2005) avec un pourcentage de la population agricole de : 79,79\% (Tone), 65,73\% (Cinkassé) et de 98,58\% (Tandjoare).

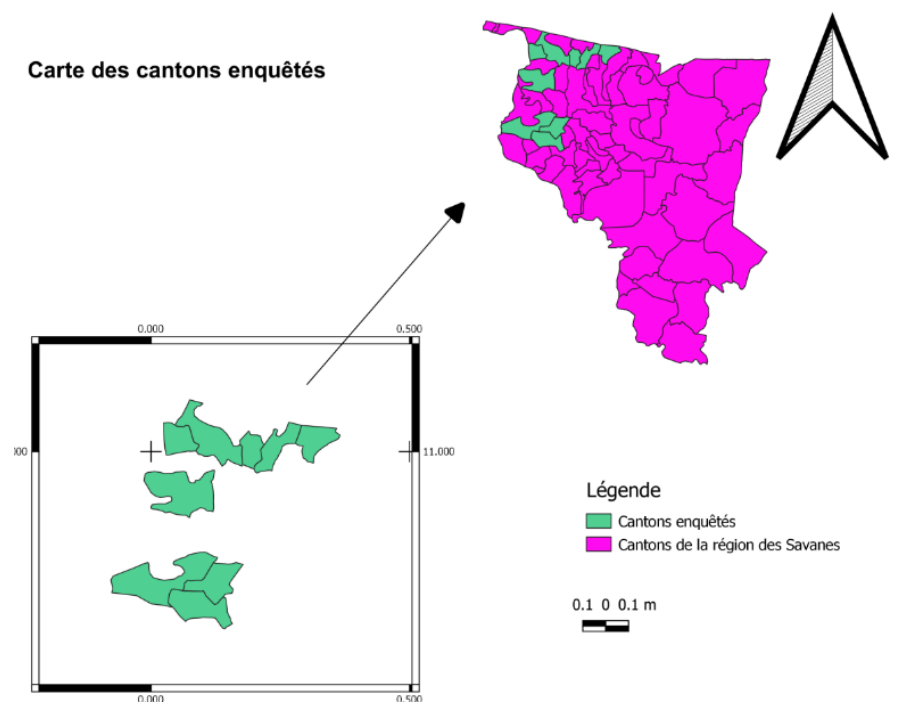

Figure 1. Localisation de la zone enquêtée 


\section{Procédure d'échantillonnage et taille de l'échantillon}

L'étude a été mené dans les cantons ayant abrité les projets « durabilité et de résilience de l'agriculture familiale dans la région des Savanes » piloté par Agronomes et Vétérinaires Sans Frontières (AVSF) et «Union Européenne » (UE) piloté par l'Institut Africain de Developpement Economique Social (INADES)-Formation. Il s'agit des cantons de :

-Timbou, Biankouri, Nadjoundi dans la préfecture de Cinkassé ;

- Sanfatoute, Naki-Ouest, Kourientré dans la préfecture de Tone ;

- Nano, Sissiak et Tampialime dans la préfecture de Tandjoare.

Dans ces cantons, 4 villages ont été choisis sur la base de l'état de dégradation des sols et de la pression foncière que subie ces villages. Dans chaque village, 6 producteurs dont 3 femmes sont choisis de façon aléatoire. Ainsi 24 producteurs ont été enquêtés par canton, soit 216 producteurs au total. La taille de l'échantillon est définie de façon raisonnée/intentionnelle en prenant en compte les ressources disponibles pour la réalisation de cette étude.

\section{Collecte de données}

Les informations sur la perception des producteurs sur la dégradation des sols, les changements climatiques et leurs conséquences, les motifs/causes d'adoption de pratiques de dégradation des sols et de l'environnement ainsi que les pratiques agro écologiques adoptées ou en cours d'adoption dans la zone ont été recueillies auprès des producteurs à l'aide d'une enquête par questionnaires. Lors de l'administration du questionnaire sur le terrain, nos propres observations ont permis de compléter les informations recueillies. Nous avons également eu des entretiens avec certains personnages œuvrant dans le domaine agricole de la région des Savanes. Les noms des espèces de plantes ont été recueillis en langues locales puis traduits en noms scientifiques par l'aide des personnes ressources.

Le dépouillement des fiches d'enquête administrées, le traitement et l'analyse des données recueillies pour interpréter les résultats.

\section{Méthode d'analyse des données}

Les statistiques descriptives, les tableaux, les graphes ont été utilisées etc. ont été utilisés pour analyser les données. La régression logistique Probit a été utilisée pour évaluer les déterminants de la perception des paysans de la dégradation des sols les dernières années d'une part, et des changements dans les températures et des cas de longues sécheresses d'autre part. Le logiciel Sphinx iQ2/EPIDATA a été utilisé pour dépouiller les questionnaires. Les données sont ensuite exportées vers les logiciels SPSS et le tableur Excel pour leur traitement et analyse.

Les résultats obtenus sont contenus dans la section suivante. 


\section{Résultats}

\section{Analyse descriptive de la population}

La population rurale des préfectures de Tône, Cinkassé et Tandjoare étaient respectivement de 91602 habitants dont 52049 femmes, 187859 habitants dont 667 femmes et 42552 habitants dont 23888 femmes (FAO, 2013). La situation matrimoniale dans ces trois préfectures des enquêtés se présente comme suit: $1,40 \%$ de personnes jamais mariés; $51,90 \%$ de monogames, $37,00 \%$ de polygames, $0,90 \%$ de divorcés ou séparés et $8,80 \%$ de veufs/veuves. L'âge de la population enquêtée est compris entre 20 ans et 90 ans avec une moyenne de 45 ans ; 29,60\% sont instruis et ont atteint les niveaux de scolarité suivants : primaire $(17,13 \%)$, collège $(1,39 \%)$, lycée $(10,65 \%)$ et programme non formel $(0,46 \%)$. Trois religions sont pratiquées par les enquêtés : le christianisme (51,40\%), l'animisme (34,30\%) et l'islam (14,40\%). L'agriculture et l'élevage sont les principales activités menées par les enquêtés avec des taux respectifs de 99,50\%. Et 0,5\%. Les activités secondaires sont l'élevage (77,80\%), le transformation (9,60\%), le commerce (0,90\%), l'artisanat (3\%), l'agriculture $(0,50 \%)$. Un seul enquêté exerce secondairement une activité de pastorat. Les enquêtés ont des ménage de 6 et 10 enfants (50,90\%), 0-5 enfants (22,70\%); 11 à 15 enfants (19,40\%), 16 à 20 enfants (5,60\%) et 21 à 25 enfants (2,30\%).

\section{Perception des producteurs sur la dégradation des sols et les changements climatiques}

La majorité des producteurs sont conscients de la dégradation de leurs sols et des changements climatiques. Les proportions perceptions des enquêtés sur ces deux évènements sont respectivement de $98,10 \%$ et $99,50 \%$. Les effets de ces deux phénomènes relevés par les producteurs sont d'ordre pluviométrique et thermique.

\section{Changements pluviométriques et thermiques}

Les changements pluviométriques se manifestent en termes de longues sécheresses, les retards de pluies, les pluies violentes, les pluies abondantes et les poches de sécheresse comme l'illustre la figure 2 suivante : 


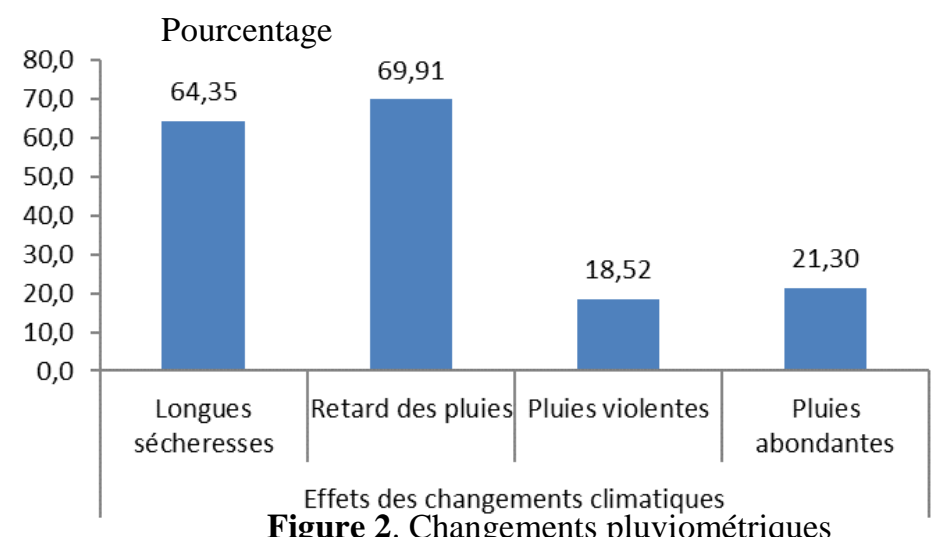

\section{Changement thermique}

Quant aux changements thermiques, elles se manifestent en termes d'augmentation ou de diminution, du non-variation ou du statu quo comme l'illustre la figure 3 :

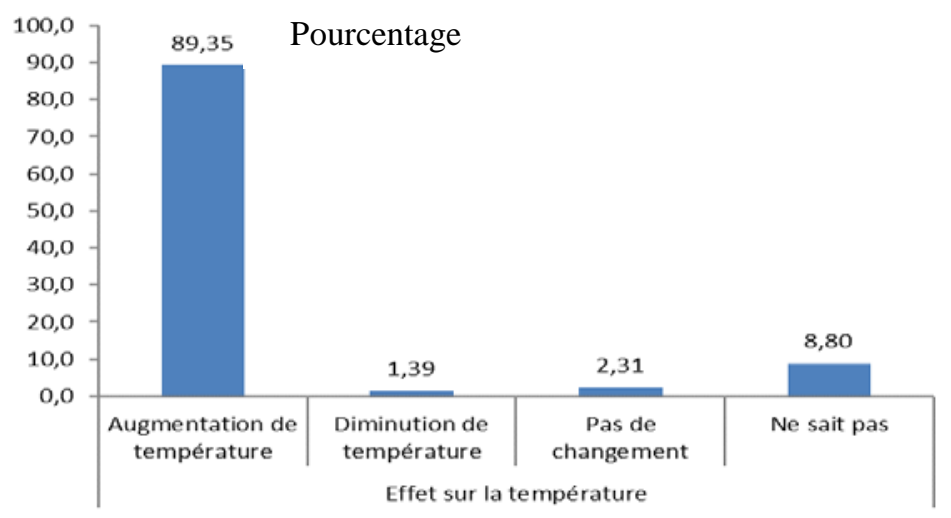

Figure 3. Perception des producteurs sur les changements thermiques

Certains producteurs sont sensibilisés sur ces changements par les ONG (22,70\%), l'Institut de Conseil et d'Appui Technique (ICAT : 13,90\%), la Nouvelle Société Cotonnière du Togo (NSCT : 1,90\%).

\section{Causes de la dégradation des sols}

Pour cette enquête 98,10\% des producteurs ont déclaré être conscients de la dégradation de leurs sols. Les causes qui sous-tendent cette dégradation ont été évoquées par ces producteurs. Pour chaque cause les producteurs ont eu à relever une ou plusieurs causes. Les proportions (nombre de personnes ayant choisi la cause/nombre totale d'enquêtés présentent comme suit : les pluies diluviennes (26,85\%), mauvaises pratiques culturales (62,50\%), intensification agricole (46,76\%), surpâturage (2,78\%), usage abusif de 
pesticides ou d'insecticides (20,37\%), l'explosion démographique (12,96\%), la déforestation (21,76\%), les feux de brousse $(6,48 \%)$, le manque de pluies (0,46\%). Il a été noté $4,63 \%$ des enquêtés qui ignorent encore les causes de la dégradation de leurs sols (figure 4). Selon leur importance, ces neuf causes peuvent être regroupées en trois dont les causes majeures, les causes moyennes et les causes mineures. Les causes majeures concernent les pluies diluviennes, mauvaises pratiques culturales, intensification agricole. Les enquêtés reconnaissent être responsables de certains de ces phénomènes soit par l'adoption de mauvaises pratiques agricoles dont la non pratique des jachères due à l'insuffisance des terres. Quant aux causes moyennes, elles sont dans l'ordre décroissant : la déforestation, l'usage abusif des pesticides/insecticides et l'explosion démographique. Enfin les feux de brousse, le surpâturage et le manque de pluie sont classée.

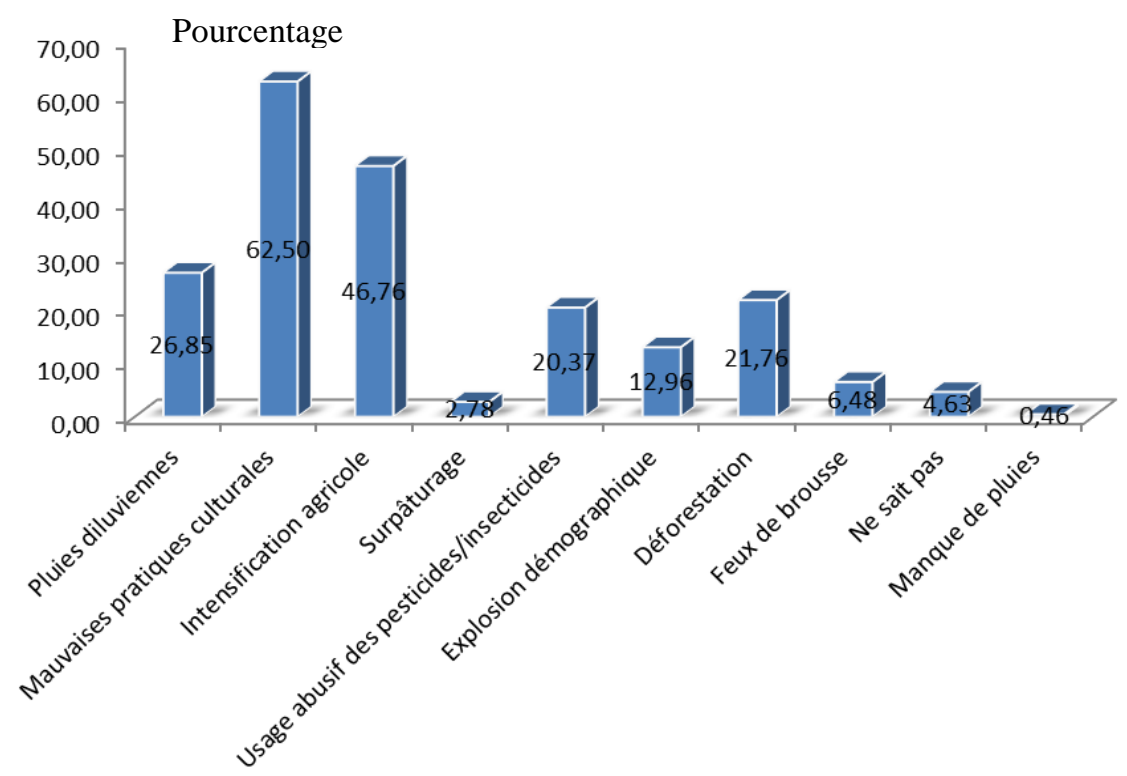

Figure 4. Proportion des causes de la dégradation des sols

\section{Régression logistique de la perception des changements dans les températures et des cas de longues sécheresses.}

Selon les résultats du tableau 1 , on peut affirmer que les variables âge du paysan, la pratique de l'élevage et l'accès aux services de vulgarisation contribuent à expliquer significativement au seuil de $10 \%$ la perception des cas de longue sécheresse par les paysans. Donc il est recommandé de prendre en compte ces variables dans les projets et programmes de luttes contre la sécheresse dans le milieu. De la même manière, les variables pratique de l'agriculture et l'accès aux services de vulgarisation sont fortement 
recommandées pour les programmes et projets de luttes contre le changement climatique dans le milieu.

Tableau 1. Régression logistique de la perception des changements dans les températures et des cas de longues sécheresses

\begin{tabular}{|c|c|c|c|c|c|c|}
\hline \multirow{2}{*}{ Variables } & \multicolumn{3}{|c|}{ Perception des cas de longues } & \multicolumn{2}{c|}{$\begin{array}{c}\text { Perception des changements } \\
\text { dans les températures }\end{array}$} \\
\cline { 2 - 7 } & $\begin{array}{c}\text { Coeffic } \\
\text { ients }\end{array}$ & $\begin{array}{c}\text { Erreurs } \\
\text { standards }\end{array}$ & $\begin{array}{c}\text { Significati } \\
\text { vité }\end{array}$ & Coefficients & $\begin{array}{c}\text { Erreurs } \\
\text { standar } \\
\text { ds }\end{array}$ & $\begin{array}{c}\text { Signifi } \\
\text { cativité }\end{array}$ \\
\hline $\begin{array}{c}\text { Sexe du } \\
\text { paysan }\end{array}$ & 0,659 & 0,678 & 0,072 & 0,421 & 0,55 & 0,584 \\
\hline $\begin{array}{c}\text { Age du } \\
\text { paysan }\end{array}$ & $-0,003$ & 0,023 & 0,804 & $-0,074$ & 0,024 & 9,488 \\
\hline $\begin{array}{c}\text { Education } \\
\text { du paysan }\end{array}$ & 0,550 & 0,679 & 0,124 & $-0,943$ & 0,607 & 2,411 \\
\hline $\begin{array}{c}\text { Pratique de } \\
\text { l'agriculture }\end{array}$ & 21,951 & 0,749 & 0,950 & 17,903 & 93,049 & 0,001 \\
\hline $\begin{array}{c}\text { Pratique de } \\
\text { l'élevage }\end{array}$ & 0,884 & 0,176 & 0,017 & 0,287 & 0,527 & 0,297 \\
\hline $\begin{array}{c}\text { Accès aux } \\
\text { services de } \\
\text { vulgarisation }\end{array}$ & 3,121 & 0,727 & 0,001 & 4,832 & 0,751 & 0,020 \\
\hline $\begin{array}{c}\text { Superficie } \\
\text { emblavée }\end{array}$ & 0,001 & 0,775 & 0,993 & $-0,071$ & 0,154 & 0,209 \\
\hline Constant & $-45,977$ & 1,214 & 0,034 & $-35,027$ & 86,097 & 0,001 \\
\hline
\end{tabular}

\section{Superficies dégradées et ampleur de la dégradation}

\section{Superficies dégradées}

. Sur 216 enquêtés, 213 soit 98,6\% ont affirmé avoir des superficies dégradées dans leur champs. Le pourcentage des enquêtés ayant des superficies se présente comme suit : $27,80 \%$ sont moins 0,50 ha ; $41,20 \%$ entre 0,5 et 1 ha; $17,10 \%$ entre 1 et 2 ha, et $12,50 \%$ des supérieures à 2 ha.

\section{Ampleur de la dégradation des sols et attitude des producteurs}

L'ampleur de dégradation des parcelles et les mesures de lutte ou de correction adoptées par les exploitants sont présentées dans cette section. Les classes d'ampleur vont du « pas du tout important » à « extrêmement important » comme résumé dans le tableau 2. Les pourcentages des classes se présentent comme suit : " pas du tout important : 2,80\% »; « Peu important : 40,70\% »; «moyennement important: 22,70\%»; « extrêmement important: 22,70\%».

Tableau 2. Ampleur de la dégradation

\begin{tabular}{|l|l|l|l|l|}
\hline $\begin{array}{l}\text { Ampleur de la } \\
\text { dégradation }\end{array}$ & $\begin{array}{l}\text { Fréque } \\
\text { nce }\end{array}$ & Pourcentage & $\begin{array}{l}\text { Pourcentage } \\
\text { valide }\end{array}$ & $\begin{array}{l}\text { Pourcent } \\
\text { age } \\
\text { cumulé }\end{array}$ \\
\hline Pas du tout important & 6 & 2,80 & 2,82 & 2,80 \\
\hline Peu important & 88 & 40,70 & 41,31 & 44,10 \\
\hline
\end{tabular}




\begin{tabular}{|l|l|l|l|l|}
\hline Moyennement important & 49 & 22,70 & 23,00 & 67,10 \\
\hline Très important & 29 & 13,40 & 13,62 & 80,80 \\
\hline Extrêmement important & 41 & 19,00 & 19,25 & 100,00 \\
\hline Total & 213 & 98,60 & 100,00 & \\
\hline Non dégradé & 3 & 1,40 & & \\
\hline Total & 216 & 100,00 & & \\
\hline
\end{tabular}

L’ampleur de la dégradation a entrainé l'abandon des parcelles dont la superficie se situé entre 37,50 ha et 63ha par 67 des producteurs soit 31,00\% des enquêtés. Les superficies abandonnées représentent en moyenne $6 \%$ des superficies cultivées par les enquêtés. Même s'il semble que les superficies abandonnées ne sont pas trop grandes, cela a un impact non négligeable sur les producteurs, étant donné qu'il s'agit ici de petits exploitants. Certains se retrouvent ainsi sans parcelle à exploiter. Cet abandon est partiel, mais dès fois total. Les superficies abandonnées sont indiquées dans le tableau 3.

Tableau 3. Superficies abandonnées

\begin{tabular}{|l|l|l|l|l|}
\hline $\begin{array}{l}\text { Superficies } \\
\text { abandonnées }\end{array}$ & Fréquence & Pourcentage & $\begin{array}{l}\text { Pourcentage } \\
\text { valide }\end{array}$ & $\begin{array}{l}\text { Pourcentage } \\
\text { cumulé }\end{array}$ \\
\hline moins de 0.5 ha & 32 & 14,80 & 47,80 & 47,80 \\
\hline 0.5 à 1 ha & 23 & 10,60 & 34,30 & 8210 \\
\hline 1 à 2 ha & 9 & 4,20 & 13,40 & 95,50 \\
\hline Plus de 2 ha & 3 & 1,40 & 4,50 & 100,00 \\
\hline Total & 67 & 31,00 & 100,00 & \\
\hline
\end{tabular}

Les résultats du tableau 4 montrent que les variables âge du paysan, mauvaises pratiques culturales, et la déforestation influencent significativement au seuil de $10 \%$ la perception de la dégradation des sols par les paysans. Ceci signifie que ce sont des variables clés à considérer dans les programmes et projets de restauration des sols dégradés dans le milieu.

Tableau 4. Régression logistique de la dégradation des sols ces dernières années dans le milieu d'étude

\begin{tabular}{|l|l|l|l|}
\hline \multirow{2}{*}{ Variables } & \multicolumn{3}{|l|}{ Perception de la dégradation des sols les dernières années } \\
\cline { 2 - 4 } & Coefficients & $\begin{array}{l}\text { Erreurs } \\
\text { standards }\end{array}$ & Significativité \\
\hline Sexe du paysan & 45,96 & 2731,055 & 0,187 \\
\hline Age du paysan & $-0,024$ & 0,051 & 0,035 \\
\hline Education du paysan & $-0,093$ & 1,882 & 0,96 \\
\hline Pluies diluviennes & 15,279 & 1042,907 & 0,588 \\
\hline Mauvaises pratiques culturales & -15.525 & 1317,711 & 0,071 \\
\hline Intensification agricole & $-76,27$ & 4304,303 & 0,986 \\
\hline Surpâturage abusif & 46,291 & 3309,587 & 0,989 \\
\hline $\begin{array}{l}\text { Usage des } \\
\text { pesticides/insecticides }\end{array}$ & 0,084 & 1,864 & 0,264 \\
\hline Explosion démographique & 30,076 & 1729,359 & 0,186 \\
\hline Déforestation & $-29,968$ & 2067,235 & 0,088 \\
\hline Constant & $-60,707$ & 3299,74 & 0,085 \\
\hline
\end{tabular}


Méthodes ou stratégies d'adaptation des producteurs face à la dégradation des sols

\section{Stratégies de lutte contre la dégradation}

Face à la dégradation, certains producteurs enquêtés se sentant impuissants ont abandonnés leurs parcelles mais d'autres adoptent des méthodes de lutte soit apprises des parents soit des organisations non gouvernementales ou encore des structures de l'Etat. Ces méthodes de lutte sont illustrées par la figure 5 .

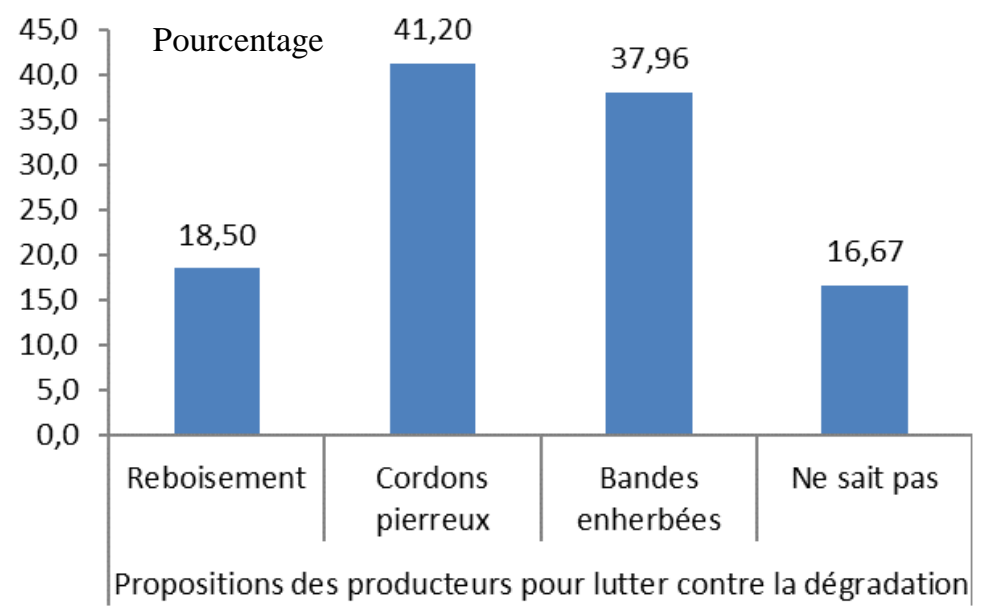

Figure 5. Stratégies de lutte contre la dégradation

\section{Cultures et pratiques culturales}

\section{Cultures}

Les producteurs de la zone d'enquête cultivent principalement les céréales (maïs: 77,30\%; sorgho/mil: 16,70\%; riz: 4,60\%; petit mil: 0,50\%) et le coton $(0,90 \%)$ comme l'indiquent le tableau 4 suivant.

Tableau 4. Cultures principales des enquêtés

\begin{tabular}{|l|l|l|l|l|}
\hline Cultures & Fréquence & Pourcentage & $\begin{array}{l}\text { Pourcentage } \\
\text { valide }\end{array}$ & $\begin{array}{l}\text { Pourcentage } \\
\text { cumulé }\end{array}$ \\
\hline Maïs & 167 & 77,30 & 77,30 & 77,30 \\
\hline Sorgho/mil & 36 & 16,70 & 16,70 & 94,00 \\
\hline Coton & 2 & 0,90 & 0,90 & 94,90 \\
\hline Petit mil & 1 & 0,50 & 0,50 & 95,40 \\
\hline Riz & 10 & 4,60 & 4,60 & 100,00 \\
\hline Total & 216 & 100,00 & 100,00 & \\
\hline
\end{tabular}

\section{Taille des superficies cultivées}

6. Dans la zone d'enquête 43,05\% exploitent des superficies inférieure à 2 ha; 34,25\% comprises entre 2 et 4 ha; 14,81\% comprises entre 4 et 6 ha; $5,55 \%$ comprises entre 6 et 10 ha et $2,31 \%$ comprises 10 et 13 ha. 


\section{Méthodes de préparation de sol et fertilisation}

Les méthodes de nettoyage du sol sont le brulis (44.9\%), simple nettoyage (52,80\%), nettoyage et brulis $(13,00 \%)$, herbicidage $(1,90 \%)$.

Le labour est effectué en billons (81,00\%) et à plat (19,00\%) par respectivement par $5,10 \%$ et $15,70 \%$ des enquêtés. Le non-labour est pratiqué par $0,50 \%$ des enquêtés.

Deux cent sept personnes soit 95,80\% utilisent les engrais chimiques tandis que $75,90 \%$ apportent la fumure organique à leurs cultures. Les doses d'engrais NPK apportées par les producteurs varient entre 0 et $300 \mathrm{~kg} / \mathrm{ha}$ pour le maïs ; 0 et $200 \mathrm{~kg} / \mathrm{ha}$ pour le mil et le sorgho, et 0 et $150 \mathrm{~kg} / \mathrm{ha}$ pour le coton. Pour l'urée, les doses sont rangées entre 0 et $200 \mathrm{~kg} / \mathrm{ha}$ pour le maïs et le sorgho, et entre 0 et $50 \mathrm{~kg}$ pour le coton. Les doses de a fumure organique se situent entre 0 et $16 \mathrm{t} / \mathrm{ha}$ pour le maïs ; 0 et $6 \mathrm{t} / \mathrm{ha}$ pour le mil/sorgho et 0 et $3,20 \mathrm{t} / \mathrm{ha}$ pour le coton. Les tableaux 5 et 6 indiquent les pourcentages des producteurs utilisant les différentes fumures sont exprimés en fonction des tranches de quantités.

Tableau 6 . Pourcentage des enquêtés utilisant le NPK et l'Urée pour le maïs, sorgho/mil

\begin{tabular}{|c|c|c|c|c|c|c|}
\hline \multirow{2}{*}{$\begin{array}{c}\text { Quantité } \\
\text { apportée }\end{array}$} & \multicolumn{2}{|c|}{ Pourcentage des enquêtés utilisant le NPK et l'Urée pour le maïs, le sorgho/mil } \\
\cline { 2 - 7 } & \multicolumn{2}{|c|}{ Maïs le coton } & \multicolumn{2}{c|}{ Sorgho/Mil } & \multicolumn{2}{c|}{ Coton } \\
\hline & NPK & Urée & NPK & Urée & NPK & Urée \\
\hline 0 & 0,9 & 5,6 & 12 & 40,7 & 0,5 & 0 \\
\hline $\begin{array}{c}0<\mathrm{Q} \leq \\
100\end{array}$ & 0,5 & 84,25 & 63,42 & 45,37 & 22,7 & 22,2 \\
\hline $\begin{array}{c}100<\mathrm{Q} \\
\leq 200\end{array}$ & 69,90 & 4,16 & 12,96 & 0,5 & 0 & 0 \\
\hline $\begin{array}{c}200<\mathrm{Q} \\
\leq 300\end{array}$ & 9,25 & 0 & 0 & 0 & 0 & 0 \\
\hline
\end{tabular}

Tableau 7. Pourcentage des enquêtés utilisant la fumure organique sous le maïs, sorgho/mil et le coton

\begin{tabular}{|l|l|l|l|}
\hline \multirow{2}{*}{$\begin{array}{l}\text { Quantité de } \\
\text { fumure organique } \\
\text { en Kg/ha }\end{array}$} & \multicolumn{4}{|l|}{$\begin{array}{l}\text { Pourcentage des enquêtés qui utilisent la fumure organique sous le } \\
\text { maïs, sorgho/mil et coton }\end{array}$} \\
\cline { 2 - 4 } & Maïs & Sorgho/Mil & Coton \\
\hline 0 & 0,5 & 23,6 & 3,2 \\
\hline $0<\mathrm{Q} \leq 1000$ & 14,81 & 13,42 & 0 \\
\hline $1000<\mathrm{Q} \leq 3000$ & 34,72 & 22,22 & 1,38 \\
\hline $3000<\mathrm{Q} \leq 5000$ & 19,44 & 4,16 & 0,5 \\
\hline $5000<\mathrm{Q} \leq 7000$ & 3,70 & 0,5 & 0 \\
\hline $7000<\mathrm{Q} \leq 10000$ & 0,5 & 0 & 0 \\
\hline $\mathrm{Q}>10000$ & 0,5 & 0 & 0 \\
\hline
\end{tabular}

Tableau 8. Rendement des cultures de maïs et de sorgho/mil

\begin{tabular}{|l|l|l|}
\hline & Pourcentage ayant ce rendement par spéculation \\
\hline Rendement (R) en t/ha & Maïs & Sorgho/Mil \\
\hline
\end{tabular}




\begin{tabular}{|l|l|l|}
\hline $\mathrm{R} \leq 1$ & 14,4 & 70,8 \\
\hline $1<\mathrm{R} \leq 2$ & 59,3 & 10,2 \\
\hline $2<\mathrm{R} \leq 3$ & 16,7 & 0,5 \\
\hline $3<\mathrm{R} \leq 4$ & 5,1 & \\
\hline
\end{tabular}

\section{Système de cultures et pratiques agro écologique}

Les méthodes d'entretien culturaux sont le sarclage/buttage à la main (86,60\%) ou avec les bœufs (27,80\%) et l'utilisation des herbicides (18,10\%). Les herbicides homologués étant rares sur les marchés locaux, les producteurs utilisent des produits tout venants disponibles, accessibles à moindre coût. Diverses pratiques agro-écologiques sont adoptées par les producteurs dans la zone. Ceux identifiées par l'enquête sont les rotations et associations culturales, les pratiques de conservation des sols, les pratiques de jachère et agroforestières.

\section{Rotation et associations culturales}

Les populations enquêtées pratiquent les rotations culturales $(86,10 \%)$ et les associations culturales $(85,20 \%)$. Les associations culturales concernent : céréales-légumineuses $(76,90 \%)$, céréales-légumes (55,60\%), céréalescéréales $(4,60 \%)$. Les rotations culturales sont : Céréales-Légumineusescéréales (31,50\%), Céréales-légumes-céréales (1,90\%), Céréales-cotoncéréales (21,80\%), Céréales-céréales (38,90\%), autres (0,90\%).

\section{Pratiques de conservation des sols}

Les méthodes de conservation des sols pratiquées par 75,90\% des producteurs. Parmi ces méthodes on note le labour suivant les courbes de niveau/ou perpendiculaire à la plus grande pente $(0,50 \%)$, Cordons pierreux (36,60\%), Diguettes en terre (12,50\%), Bandes anti-érosives (50,90\%), autres $(2,30 \%)$. Les pratiques sont découvertes par l'intermédiaire des ONG (19,00\%), des services techniques de l'Etat $(7,40 \%)$ ou transmises par les parents/initiatives personnelles (52,80\%). Cependant certains producteurs ne les utilisent pas. Les raisons évoquées sont leur fastidiosité (8,80\%), la méconnaissance de leur importance (13,40\%), et leur cout élevé $(2,80 \%)$.

\section{Pratiques de jachère, agroforesterie et plantation d'arbres}

Les jachères sont pratiquées par 1,90\% des enquêtés. L'insuffisance des terres (98,10\% des enquêtés) est la principale cause de ces courtes jachères de durée maximum de 3 ans.

L'utilité ou les motifs/raisons de plantation des arbres par les enquêtés sont relevés dans le tableau 5 . Les producteurs s'adonnent à deux pratiques agroforestières : les plantations individuelles $(84,30 \%)$ ou collectives $(1,90 \%)$ et la régénération naturelle assistée (42,10\%). Les superficies plantées sont 
variables : 82,40\% des producteurs ont plantés sur moins de $25 \%$ des superficies, $1,40 \%$ sur des superficies situées entre 25 et $50 \%$, et $0,50 \%$ sur 50 à $100 \%$ des superficies exploitées. Au total 181 producteurs soit $83,8 \%$ des enquêtés plantent 2450 plants/an sur une superficie de 660 ha ce qui équivaut en moyenne à 4 plants/ha/an. Le risque de mort de plants pour raisons de surpâturage ou de rudesse de la longue sèche, le nombre de plants survivants/ha/an serait en deçà de ce chiffre.

Tableau 9. Raisons de plantation des arbres

\begin{tabular}{|l|l|}
\hline Raisons de la plantation & Pourcentage (\%) \\
\hline Ombre & 88,00 \\
\hline Fruit & 85,60 \\
\hline Fourrage & 7,90 \\
\hline Feuilles & 5,10 \\
\hline Fertilisation des sols & 42,10 \\
\hline Bois de chauffe & 85,20 \\
\hline Vente, commerce & 16,70 \\
\hline Vertu médicinale & 51,90 \\
\hline
\end{tabular}

Certains enquêtés ont évoqué des difficultés à se procurer de plantules (8,30\%), le cout élevé du travail (6,90\%) et la manière fastidieuse de faire ce travail $(2,30 \%)$.

\section{Espèces de plantes désirées par les producteurs}

Les principales espèces de plantes voulues par les producteurs sont illustrées par la figure 17. L'espèce la plus désirée est Erythrina senegalensis (99,10\%); elle est suivie par six (6) espèces moyennement désirées : Azadiratcha indica (77,30\%), Manguifera indica (72,20\%), Vitellia paradoxa $(70,40 \%)$, Parkia biglobosa $(70,40 \%)$, Cacia siamea $(50,50 \%)$, Borassus aethiopum $(48,10 \%)$ et six (6) espèces modérément désirées: Ecalyptus globulus (35,60\%), Kaya senegalensis (33,80\%), Citrus sinensis (18,10\%), Prosopis africana (14,80\%) Moringa oleifera (13,90\%), Anarcadium occidentale (13,00\%). Pour les autres espèces le pourcentage de producteurs les désirant est moins de $10 \%$.

La figure 6 illustre le pourcentage des producteurs ayant reconnu l'existence actuelle des espèces végétales. Les espèces les plus répandues sont: Azadiratcha indica (75,90\%), Manguifera indica (72,70\%), Vitellia paradoxa (63,90\%), Parkia biglobosa (62,00\%), Cacia siamea (50,00\%), Borassus aethiopum (41,70\%), Ecalyptus globulus (34,70\%), Kaya senegalensis (29,20\%), Citrus sinensis (19,00\%), Prosopis africana (13,00\%) Moringa oleifera (13,40\%), Anarcadium occidentale (12,50\%). Par contre Erythrina senegalensis, l'espèce la plus désirée a presque disparue dans la zone. Seulement 6 enquêtés sur 216 confirme l'existence de cette plante. 


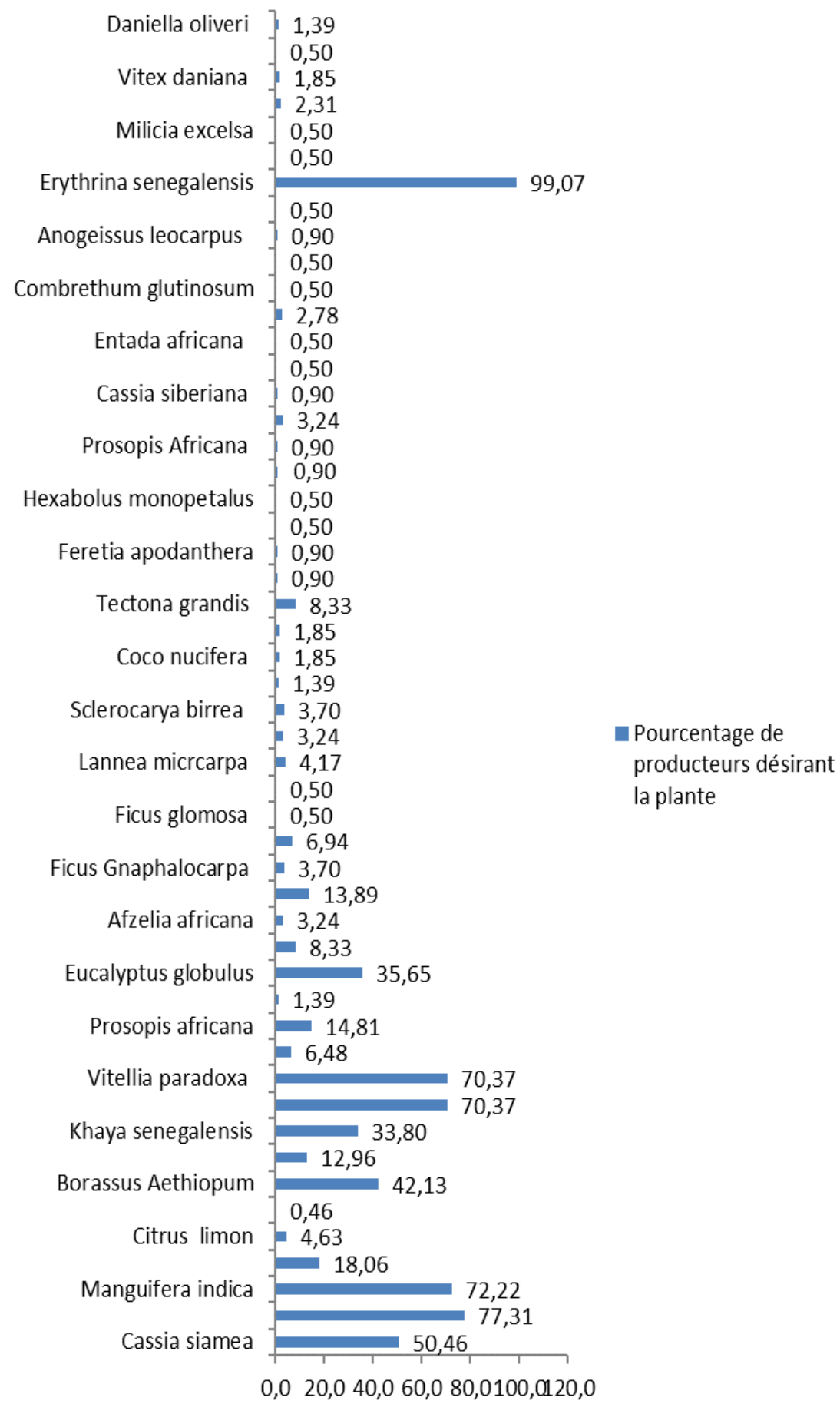

Figure 6. Plantes désirées par les producteurs 


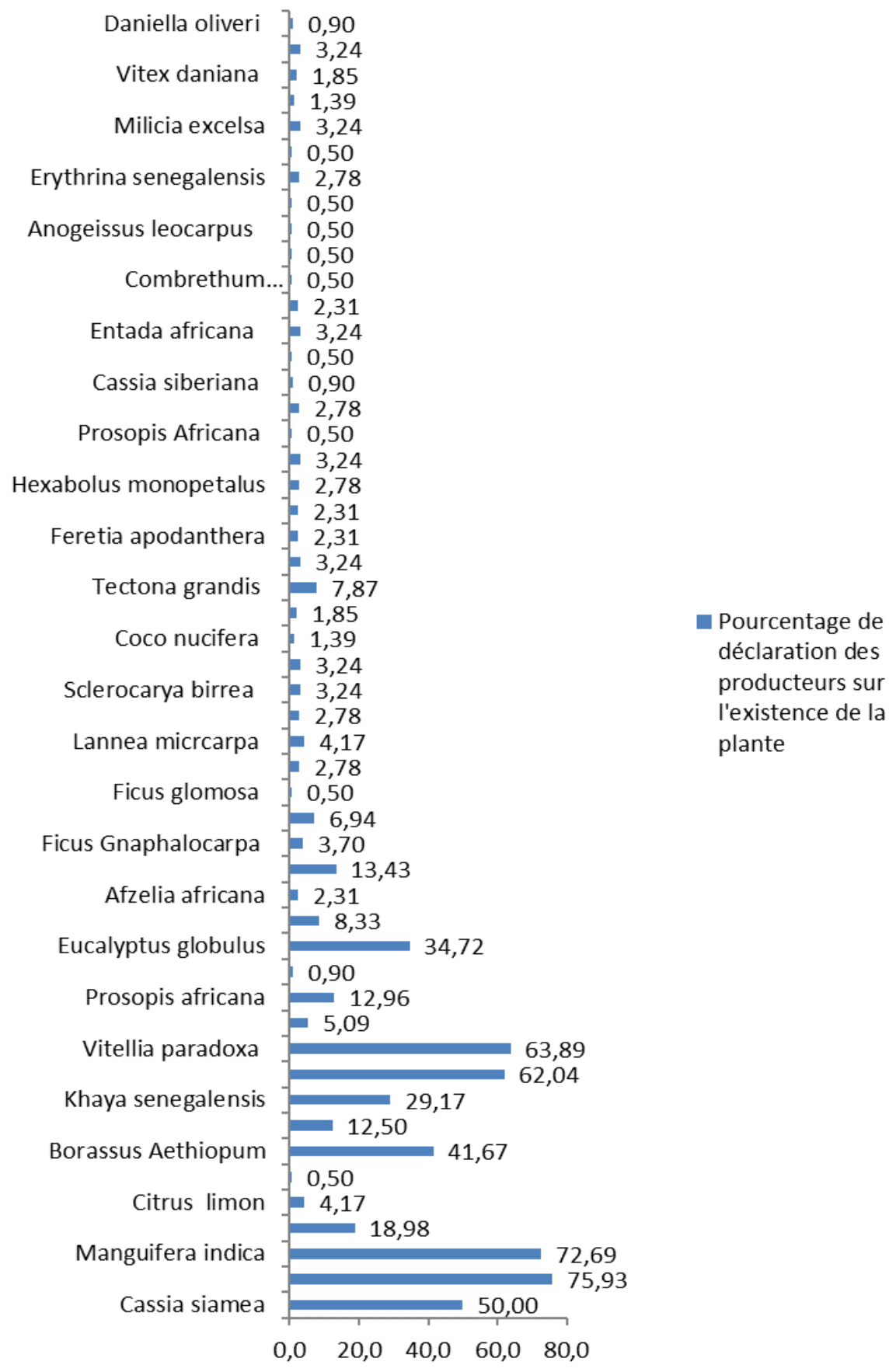

Figure 7. Existence de la plante 


\section{Croisement espèces les plus désirées et son existence}

La figure 8 situe sur la régression/disparition des espèces les plus désirées. L’espèce Erythrina senegalensis plus désirée est en forte régression ainsi que Vitellia paradoxa, Parkia biglobosa, Khaya senegalensis.

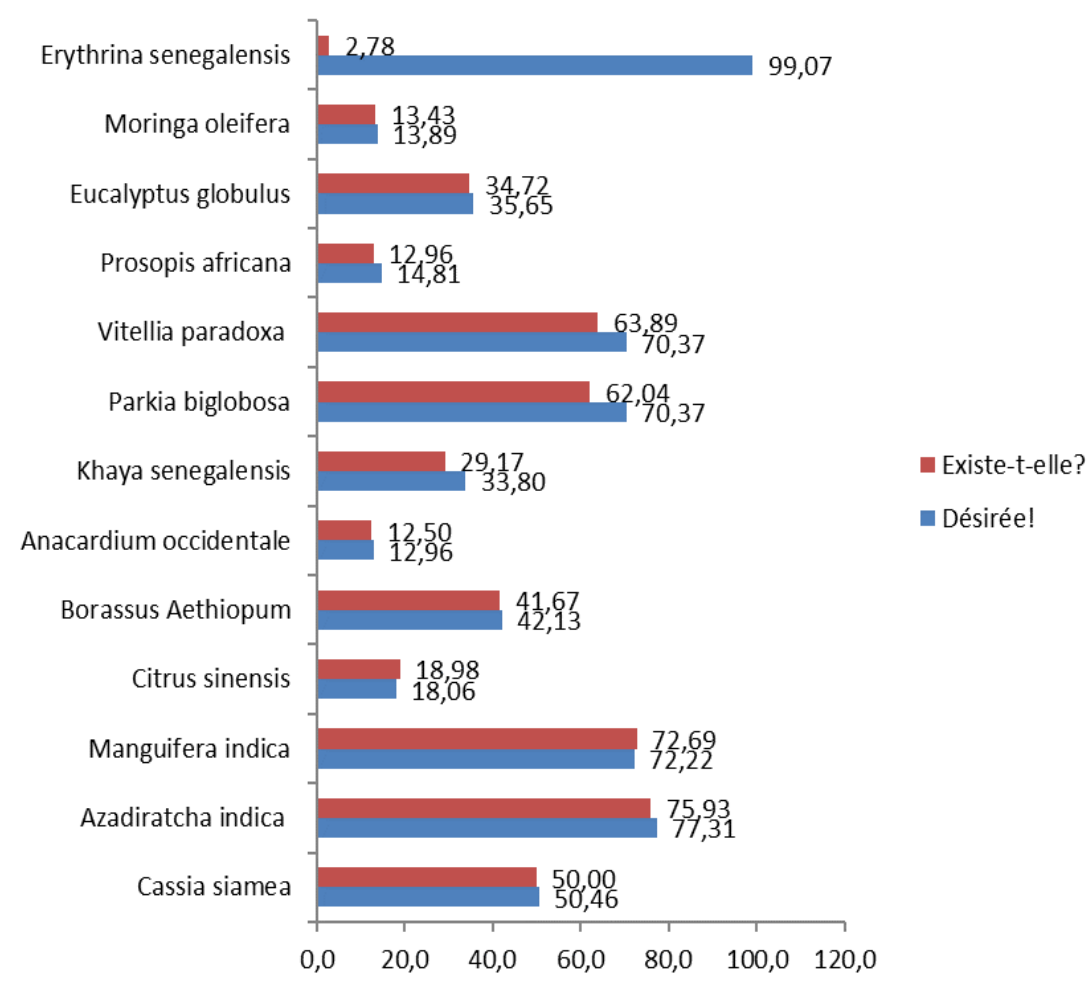

Figure 8. Existence des espèces désirées par les producteurs

\section{Gestion des résidus de récoltes}

Les résidus de récolte sont: (i) utilisés comme source d'énergie (80,10\%) (ii) l'aliment du bétail/fourrage (17,10\%) (iii) pour la construction des palissades (8,80\%), (iv) abandonnés au champ (36,10\%) ou (v) brulés $(6,50 \%)$.

\section{Discussion}

Perception des producteurs sur la dégradation des sols et les changements climatiques

Parlant d'abord du niveau d'instructions des enquêtés, 29,60\% seulement ont été instruis. Cette proportion est supérieure au résultat de Soviadan (2015) qui avait révélé seulement un taux de scolarisation de 23,23\% lors d'une enquête réalisée sur les cotonculteurs de la région de la Savane.

Les effets des changements climatiques relevés par les producteurs sont les retards de pluies, les pluies violentes, les pluies abondantes et les poches 
de sécheresse. Pour les changements thermiques, elles se manifestent selon la majorité des enquêtés par en termes d'augmentation de températures ces 10 dernières années. La majorité des enquêtés ont relevés une augmentation des superficies dégradées et des températures se confirme l'étude de Sanou et al. (2018). Les conséquences de cette augmentation sont entre autres la diminution des ressources naturelles, la baisse des rendements, les difficultés d'abreuvement des animaux, l'accélération de la dégradation des sols. Les scénarios prédits par (MERF, 2015) concernant la zone d'étude font état d'une augmentation des températures qui auront des incidences sur les cultures maraîchères. Ces résultats corroborent l'étude d'Adetou et Ahlin (2019) qui ont montré que la plupart des producteurs de la région des Savanes pensent que les conditions climatiques des 10 dernières années se sont dégradées. Selon le même auteur, plus de la moitié (55\%) des togolais ont une connaissance des changements climatiques contre $44 \%$ qui les ignorent. La proportion de la population ayant connaissance de ces changements dans la zone reste élevée (99,50\%) ce qui peut s’expliquer par les récents événements d'inondation. Cette proportion laisse voir que tant les hommes que les femmes connaissance du changement climatique contrairement à l'étude de World Bank (2018) selon laquelle, les femmes ont moins connaissance des changements climatiques que les hommes bien qu'elles y participent à ces changements par leurs activités de prélèvement de bois de cuisine et de plantes médicinales. Ce qui s'explique par la faible proportion de femmes ayant une éducation formelle.

Les pluies violentes et abondantes relevées par les enquêtés sont une confirmation des études et événements précédents. En effet, la région a connu des phénomènes extrêmes à l'instar des inondations de 2007 et 2013 qui ont causés des dégâts lourds et des pertes en vies humaines (Adewi, 2010 ; UNISDR, 2013). La dégradation des sols est partout visible par la présence de ravines et de l'ensablement des cours d'eau rencontré partout dans la zone.

\section{Causes et ampleur de la dégradation des sols}

Les producteurs ont relevé les causes de la dégradation des sols de la zone classée parmi les plus dégradées par Brabant en 1996. Neuf causes sont évoquées à savoir : les pluies diluviennes, mauvaises pratiques culturales, intensification agricole, surpâturage, usage abusif de pesticides ou d'insecticides, l'explosion démographique, la déforestation, les feux de brousse, le manque de pluies. Les neuf causes se regroupent en deux causes souvent citées dans la littérature à savoir les causes biophysiques, les causes socioéconomiques et politiques et des impacts des changements climatiques ou anthropiques (Gueye, 2013; Benslimane al., 2008 et Hien, 1998). L'accroissement de la population et la pauvreté sont cités parmi les causes dégradation des sols dans le monde par Chirwa (2014); UNCCD (2015). En 
effet, le nord-ouest de la région des Savanes est la plus peuplée avec une densité de $213 \mathrm{hbts} / \mathrm{km}^{2}$ comparée à la moyenne régionale de $98 \mathrm{hbts} / \mathrm{km}^{2}$ (RGPH, 2010). Cette population concentrée exerce une pression sur les ressources naturelles ce qui a entrainé la disparition de la couverture végétale et de la jachère (Vieira et al., 2015; Bareremna Afelu et al., 2016). Les sols dénudés par la déforestation et les feux de brousse sont ainsi exposés aux pluies souvent très violentes et à l'érosion éolienne et hydrique (Pitot, 1952). Sur les sols dénudés et pâturés, les coefficients de ruissellement, l'érosion de sol arbre, les densités apparentes et la conductibilité hydraulique sont plus élevé comparé aux sols couverts d'arbre et non surpâturés (Viramontes, 2000; Cosandey, 1995; Fritsch, 1995; CILSS, 2011.). Dans cette zone la réticence des producteurs à adopter les bonnes pratiques culturales et la divagation des animaux en saison sèche viennent envenimer la situation (N'guessan et al., 2015). Dans la plupart des cas, la dégradation des sols est aggravée par la méconnaissance de l'utilisation rationnelles des sols (Omar et al., 2013. Bhattacharyya et al., 2015). Les mauvaises pratiques culturales et l'utilisation des herbicides sont connus comme ayant un effet néfaste sur les sols (Chitrit, 2011;). Les mauvaises pratiques culturales concernent à la fois le mauvais travail du sol et une mauvaise gestion des cultures. Naitormbaide et al. (2010) ont montré que certains systèmes de cultures favorisent la dégradation des sols et les stocks de carbone. Cette étude confirme les travaux de d'Adetou et Ahlin (2019) où les agriculteurs de la région des Savanes indexent les activités humaines comme étant la principale cause des changements climatiques.

Le nombre des enquêtés ayant des parcelles dégradées $(98,60 \%)$ confirme les travaux de Brabant (1996) qui avait classé cette zone parmi les plus dégradées du territoire togolais. Les proportions des enquêtés dont les dégradations des sols sont " moyennement importantes » et " extrêmement importantes » sont respectivement de $22,70 \%$ et $22,70 \%$.

\section{Méthodes d'adaptation à la dégradation des sols et les pratiques agro écologiques}

Pour lutter contre la dégradation des sols, les producteurs font recours à certaines pratiques agro écologiques notamment le reboisement, des mesures de conservation des sols comme la mise en place des cordons pierreux, et des bandes enherbées, les rotations et associations culturales et l'apport de la fumure organique (Levard et Mathieu, 2018). De telles stratégies d'adaptations étaient adoptées depuis longtemps par les producteurs de Boulgou au Burkina Faso (Hien, 1998). Ces pratiques sont promues dans la zone par des ONG et des services étatiques comme l'institut Togolais de Recherche Agronomique (ITRA) (Levard et Mathieu, 2018). Cependant beaucoup utilisent encore des méthodes aggravantes de la dégradation comme le brulis, le labour en billons dans le sens de la pente. Les raisons évoquées 
par les producteurs en ce qui concerne la faible adoption de ces pratiques sont entre autres leur pénibilité et le manque de moyens financiers.

Les résultats de l'enquête montrent que la matière organique est apportée en très faibles quantité alors que les cultures principales étant les céréales nécessitent obligatoirement l'apport des engrais chimiques pour espérer avoir une récolte ce qui explique la proportion très élevée des ceux qui utilisent des engrais chimiques. Les associations et les rotations de cultures pratiquées n’ont pas d'effet remarquable sur la fertilité du sol étant donné que les résidus de résidus de récolte sont exportés soit pour l'alimentation du bétail ou encore pour en faire des combustions. En 2003, Djagni signalait le nonrespect des recommandations de la vulgarisation sur les parcelles cultivées en coton où les résidus de récolte. La restauration de ces terres doit passer obligatoirement par l'augmentation des quantités de matière organique. L'adoption progressive de certaines méthodes de conservation des sols telles que la mise en place des bandes anti-érosives, des cordons pierreux, des diguettes en terre sont initiées par les ONG et les structures d'Etat. Ces pratiques permettent non seulement de récupérer les sols, mais également d'accroître leur fertilité et le rendement des cultures ; une expérimentation menée par Dapola Da (2008) a montré que les méthodes de conservation des sols améliorent les rendements du sorgho. En effet les rendements du sorgho sur les parcelles aménagées furent nettement supérieures au témoin : zaï $(1,70$ t/ha); les bandes enherbées d'Andropogon gayanus (1,25 t/ha); cordons pierreux $(0,84 \mathrm{t} / \mathrm{ha})$; paillage $(0,77 \mathrm{t} / \mathrm{ha})$ contre $0,12 \mathrm{t} / \mathrm{ha}$ pour témoin, sans méthode de conservation.

\section{Pratiques agroforestières et espèces végétales préférées par les producteurs}

Les pratiques agroforestières mis en place par les exploitants sont les plantations individuelles collectives des arbres et la régénération naturelle assistée. Les espèces plantées sont pour la plupart des essences ayant des vertus multiples. Pour plus de $50 \%$ des producteurs, le choix de l'espèce dépend de sa capacité à fournir de l'ombre, à fournir des fruits pour la consommation humaine et à soigner (vertu médicinales). Des résultats voisins ont été notifiés par Levard et Mathieu (2018) au Burkina Faso où 100\% des producteurs préfèrent reboiser des espèces pour leur ombre, leurs racines, leurs feuilles, leurs fruits et leur tronc. Les espèces comme Erythrina senegalensis, Vitellia paradoxa, Parkia biglobosa, Khaya senegalensis sont bien préférées par les producteurs à cause de leurs diverses vertus mais en forte régression (Akpagana, 2006). 


\section{Conclusion}

Le nord-ouest de la région des Savanes est une zone très dégradée et sujette aux aléas climatiques et effets anthropiques. L’enquête a montré que certains producteurs conscients de la situation adoptent des méthodes de résilience alors que d'autres restent encore inconscients. Les pratiques culturales préservatrices des sols telles que la rotation et l'association des cultures, le reboisement, les mesures de conservation des sols sont observées par certains producteurs dans un contexte de pression démographique où les jachères jadis pratiquées par les producteurs sont quasi inexistantes. Certaines espèces végétales sont diversement appréciées par les producteurs. Les producteurs impuissants ont purement et simplement abandonné une partie de leur parcelle qu'ils estiment irrécupérable. A l'issu de cette étude, les conclusions suivantes peuvent être tirées :

-la dégradation des sols et de l'environnement due à l'explosion démographique et aux changements climatiques sont connus par la plupart des producteurs.

-la dégradation a entrainé l'abandon des parcelles par certains producteurs ;

-Certains producteurs adoptent des méthodes de résiliences telles que les pratiques agro écologiques, des méthodes de conservation des sols et de reboisement ;

-les espèces végétales préférées par les producteurs ont disparues ou sont en voie de disparition d’où la nécessité de les préserver et d’insérer dans les programmes de reboisement

Une étude économique des ménages est souhaitée afin de déterminer les pertes causées par cette situation et les gains des producteurs qui adoptent des méthodes de résilience. Il sera également intéressant de faire une étude poussée sur les préférences de plantes et leur capacité de stocker le carbone du sol.

\section{References :}

1. Adetou A. E. et Ahlin E. A., 2019. Les changements climatiques empirent la vie au Togo mais sont moins connus par les agriculteurs. Dépêche No. 279, Afrobaromètre, 11p.

2. Adewi E., Dubreuil V. et Badameli K.M.S., 2010. Instabilité pluviométrique dans la région des Savanes à l'extrême nord du Togo. Actes du 23ième Colloque de l'Association Internationale de Climatologie, pp 35-40.

3. Akpagana K., 2006. Savoirs locaux et gestion de la biodiversité : Habitudes alimentaires et utilisation des plantes alimentaires mineures ou menacées de disparition au Togo. Rapport technique Année III. 13 Avril 2006 
4. Annette F., Mark D.A. R., Pete S. et Jan V., 2004. Carbon sequestration in the agricultural soils of Europe. 24p.

5. Bank. 2018. The World Bank Annual Report 2018 (English). Washington, D.C. World Bank Group. http://documents.worldbank.org/curated/en/630671538158537244/Th e-World-Bank-Annual-Report-2018.

6. Bareremna A., Fontodji K. J. et Kokou K., «Impact des feux sur la biomasse dans les savanes guinéo-soudaniennes du Togo », VertigO la revue électronique en sciences de l'environnement [En ligne], Volume 16 Numéro 1 | mai 2016, mis en ligne le 09 mai 2016, consulté le 21 janvier 2020. URL : http://journals.openedition.org/vertigo/17106 ;

DOI : 10.4000/vertigo.17106

7. Benslimane1, M., A. Hamimed, W. El Zerey, A. Khaldi, K. Mederbal, 2008. Analyse et suivi du phénomène de la désertification en Algérie du nord, VertigO - La revue en sciences de l'environnement, Vol8 no3, 9p.

8. Brabant P., Simonneaux V., Egue K., 1994. Etat actuel de dégradation des terres et de l'environnement au Togo déterminé à l'aide de la télédétection. P 169-188.

9. Chitrit J. J., Gautronneau Y., Pratiques agricoles et fertilité des sols en France, Revue Science Eaux \& Territoires, article hors-série, 8 p., 01/01/2011, disponible en ligne sur <URL : http://www.setrevue.fr/pratiques-agricoles-et-fertilite-des-sols-en-france> (consulté le 29/01/2020), DOI : 10.14758/SET-REVUE.2011.HS.01

10. Dapola Da C. E., «Impact des techniques de conservation des eaux et des sols sur le rendement du sorgho au centre-nord du Burkina Faso », Les Cahiers d'Outre-Mer [En ligne], 241-242 | Janvier-Juin 2008, mis en ligne le 01 janvier 2011, consulté le 11 février 2020. URL : http://journals.openedition.org/com/3512 ;

DOI : https://doi.org/10.4000/com.3512

11. Chirwa P.W., 2014. Restoration practices in degraded landscapes of Southern Africa. African Forest Forum, Working Paper Series, 12 (2), $44 \mathrm{p}$.

12. Comité permanent Inter- États de Lutte contre la Sécheresse dans le Sahel (CILSS), 2011. Comment récupérer les terres totalement dégradées et dénudées ou "ZIPELLE » par la technique du tapis herbacé. 1-20.

13. Cosandey C., 1995. La forêt réduit-elle l'écoulement annuel ? Annales de Géographie, $n^{\circ}$ 581- 582, pp. 7-25.

14. Djagni K. K., 2003. L'agriculture togolaise face à des mutations environnementales multiples: nécessité d'un ensemble d'innovations 
techniques et organisationnelles cohérentes; necessity of a coherent package of technical and organisational innovations. 9 p. hal00128887

15. FAO ; 2013. 4ème recensement national de l'agriculture 2011-2014, module II : module de base; résultats chiffrés et détaillés de l'agriculture togolaise.

16. FAO, 2005. Appui à la mise en œuvre du NEPAD-PDDAA. Projet de gestion intégrée de la fertilité des sols et sécurisation foncière. 32p.

17. Fritsch J. M., Occupation des sols et régimes hydrologiques : les enseignements des bassins versants guyanais. In: Annales de Géographie, t. 104, n581-582, 1995. pp. 26-63; doi:https://doi.org/10.3406/geo.1995.13867 https://www.persee.fr/doc/geo_00034010_1995_num_104_581_1386 7

18. Gueye I., 2013. Application de la Télédétection aérospatiale pour l'évaluation de la dégradation des ressources naturelles : cas des sols de la région de Kaolack située dans le Bassin arachidier du Sénégal, mémoire de master CB2A, UCAD, 148p.

19. Hien S. A., 1998. La perception paysanne de la dégradation de l'environnement et les stratégies d'adaptation: cas de Niaogho Beguedo dans la province de Boulgou. Mémoire de maitrise. Université de Ouagadougou, département de géographie, 152p.

20. Levard L., Mathieu B., 2018. Agroécologie : capitalisation d'expériences en Afrique de l'Ouest. Facteurs favorables et limitants au développement de pratiques agroécologiques. Evaluation des effets socio-économiques et agro-environnementaux. Document de capitalisation CALAO, CEDEAO-AFD. 80p.

21. MERF (Ministère de l'Environnement et des Ressources Forestières) ; 2015. Contribution prévue déterminée au niveau national (CPDN) dans le cadre de la convention cadre des nations unies sur les changements climatiques (CCNUCC). 21p.

22. MERF, 2015. Troisième communication nationale du Togo sur les changements climatiques 136p.

23. Naitormbaide M., Lompo F., Gnankambary Z., Ouandaogo N. et Sedogo M. P. August 2010. Les pratiques culturales traditionnelles appauvrissent les sols en zone des savanes du Tchad. Int. J. Biol. Chem. Sci. 4(4): 871-881.

24. N'guessan K. A., Diarrassouba N., Alui K. A., Nangha K.Y., Fofana I. J., \& Kouame A. Y., 2015. Indicateurs de dégradation physique des sols dans le Nord de la Côte d'Ivoire : cas de Boundiali et Ferkessédougou. Afrique SCIENCE 11(3) 115 - 128. 
25. Omar A. A., Edinam K. G. \& Olavi L., 2013. Causes and Impacts of Land Degradation and Desertification: Case Study of the Sudan. International Journal of Agriculture and Forestry, 3(2): 40-51

26. Pitot A., 1952. L'homme et les sols dans les steppes et savanes d'A.O.F. Les cahiers d'Outre-Mer, pp 215-240.

27. Poulenard J., Descroix., Janeau J. L., 1996. Surpâturage et formation des terrassettes sur le versant de la Sierra Léone Madre occidentale (Nord-Ouest du Mexique). Revue Géographie Alpine. 84.

28. Sanou K.; Amadou S.; Adjegan K.; Tsatsu K.D., 2018. Perceptions et stratégies d'adaptation des producteurs agricoles aux changements climatiques au nord-ouest de la région des savanes du Togo. Agronomie Africaine ; pp 87-97.

29. Soviadan M. K., 2016. Impact des changements climatiques sur la production du coton-graine : cas de la Région des Savanes, NordTogo.126p.

30. UNISDR, 2013. Rapport d'évaluation sur l'intégration et la mise en œuvre des mesures de réduction des risques de catastrophe au Togo ; $76 \mathrm{p}$

31. United Nations Convention to Combat Desertification (UNCCD), 2015. Climate change and land degradation: Bridging knowledge and stakeholders. 9-12 March 2015, Cancún, Mexico; Outcomes from the UNCCD 3rd Scientific Conference; 19pp.

32. Vieira, D.C.S., C. Fernandez, J.A Vega et J.-J. Keizer, 2015. Does soil burn severity affect the post-fire runoff and erosion response? a review based on meta-analysis of field rainfall simulation data, Journal of Hydrology, Volume 523, 15, pp. 452-464.

DOI : 10.1016/j.jhydrol.2015.01.071

33. Viramontes D., Descroix L. Dégradation progressive du milieu par le surpâturage et le déboisement et conséquences hydrologiques. Étude de cas dans la Sierra Madré occidentale (Mexique). In: Revue de géographie alpine, tome $88, \mathrm{n}^{\circ} 2$, 2000. pp. 27-41; doi:https://doi.org/10.3406/rga.2000.2989; https://www.persee.fr/doc/rga_0035-1121_2000_num_88_2000 